\title{
Improved measurement linearity and precision for AMCW time-of-flight range imaging cameras
}

\author{
Andrew D. Payne, ${ }^{1, \star}$ Adrian A. Dorrington, ${ }^{1}$ Michael J. Cree, ${ }^{1}$ and Dale A. Carnegie ${ }^{2}$ \\ ${ }^{1}$ School of Engineering, University of Waikato, Private Bag 3105, Hamilton, New Zealand \\ ${ }^{2}$ School of Engineering and Computer Science, Victoria University of Wellington, \\ P.O. Box 600, Wellington, New Zealand \\ *Corresponding author: a.payne@ waikato.ac.nz
}

Received 11 March 2010; revised 7 July 2010; accepted 8 July 2010; posted 13 July 2010 (Doc. ID 125268); published 5 August 2010

\begin{abstract}
Time-of-flight range imaging systems utilizing the amplitude modulated continuous wave (AMCW) technique often suffer from measurement nonlinearity due to the presence of aliased harmonics within the amplitude modulation signals. Typically a calibration is performed to correct these errors. We demonstrate an alternative phase encoding approach that attenuates the harmonics during the sampling process, thereby improving measurement linearity in the raw measurements. This mitigates the need to measure the system's response or calibrate for environmental changes. In conjunction with improved linearity, we demonstrate that measurement precision can also be increased by reducing the duty cycle of the amplitude modulated illumination source (while maintaining overall illumination power). (C) 2010 Optical Society of America
\end{abstract}

OCIS codes: $\quad 110.6880,150.5670,280.3640,150.6910,120.2920,120.5050$.

\section{Introduction}

Time-of-flight range imaging is an emerging technology [1-11] that produces a "distance image" of a scene, where each pixel in the image not only contains intensity information, but also the range (or distance) from the camera to the object. A threedimensional reconstruction of the scene can be generated from the captured data, providing the opportunity to measure the location, size, and shape of objects-attributes that are highly desirable in a wide array of applications, such as machine vision, surveying, medical imaging, surface profiling, and human-machine interaction.

Range images are produced by illuminating the scene with an amplitude modulated continuous wave (AMCW) light source, typically a bank of nearinfrared light emitting diodes (LEDs). The light travels to, and is reflected back from, objects in the scene with a propagation time proportional to the

0003-6935/10/234392-12\$15.00/0

(C) 2010 Optical Society of America distance traveled. This propagation time manifests as a phase shift of the illumination modulation envelope. A specialized gain modulated image sensor then samples the reflected light and measures the phase shift at every pixel to produce a range image of the scene. As independent phase measurements are performed in parallel for each pixel, the system can provide range data of an entire scene at rapid (video) update rates.

The amplitude modulation waveform produced by the LED illumination source is typically nonsinusoidal and, therefore, contains a number of higher-order harmonics that are aliased during the sampling process at the image sensor, resulting in a nonlinear phase (and, therefore, range) measurement [1]. The typical approach used to correct the linearity error is to calibrate the system by measuring the system response [1-5]; the error is then subtracted during postprocessing. Performing a calibration is a timeconsuming task, and if any system parameters are altered, the calibration will likely need to be repeated.

An alternative method of removing the systematic range linearity error is presented, where harmonic 
components in the illumination (amplitude modulated) waveform are canceled by the image sensor during the sampling process. The technique can be applied to existing range imaging systems without requiring modification of the specialized image sensor or data processing stages, instead only requiring an adaptation of the signal generation stage. By canceling the harmonic components during sampling, the resultant system calibration is simplified, and robustness due to parameter variation, such as manufacturing and environmental variations, is improved.

\section{AMCW Time-of-Flight Range Imaging}

A range image is produced by flood-illuminating an entire scene and measuring the time of flight (or propagation delay) of the light as it is reflected from objects back to an image sensor. The flight time is given by $2 r / c$ where $r$ is the object range, $c$ is the speed of light, and the factor of 2 allows for the round-trip distance traveled both to and from the target. For $\mathrm{AMCW}$ systems, an indirect measurement is performed where the illumination source is amplitude modulated and the propagation delay is manifested as a phase shift of the modulation envelope of the reflected light. A specialized image sensor synchronously samples the reflected light by modulating the gain of each pixel at the same frequency as that of the illumination source (homodyne detection), integrating the result over an integration period. The integration period allows a significant number (tens of thousands) of modulation cycles to be combined to achieve a high signal-to-noise ratio (SNR) before reading the output from the sensor, where the output voltage represents a correlation between the received illumination and the sensor modulation signals.

For example, if an object is located near the camera, the phase shift of the modulation envelope will be small, producing a strong correlation between the illumination and the image sensor modulation waveforms, which is seen as a bright pixel value. Conversely, the illumination and sensor modulation waveforms are out of phase for distant objects, producing dull pixel values. The pixel intensity is not only dependent on the phase shift due to the propagation time, but is also affected by object surface properties (color, reflectivity, etc.), as well as ambient lighting. To independently resolve the modulation envelope phase (as well as the amplitude and offset parameters), the correlation measurement is repeated $N$ times with a phase step of $2 \pi j / N$ rad introduced to the sensor or illumination modulation signal, where $j=0,1,2, \ldots, N-1$, producing intensity values $I_{0}, \ldots I_{N-1}$ as illustrated in Fig. 1 . A typical value of $N=4$ has been selected for the illustration in Fig. 1, while the correlation function has been generated using an ideal illumination sinusoidal amplitude modulation response.

From the $N$ intensity values, the amplitude $A$, phase $\varphi$, and offset $B$ of the correlation function can then be determined for each pixel by

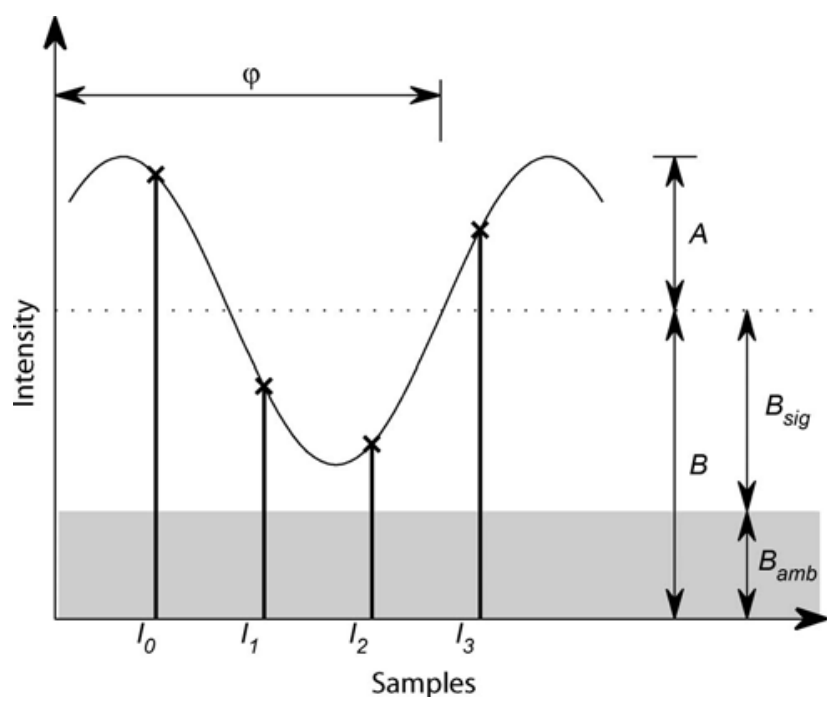

Fig. 1. Image sensor synchronously samples the reflected illumination, performing a correlation between the amplitude modulated illumination and the amplitude modulated sensor gain. Four correlation measurements are collected to allow the signal amplitude $A$, phase $\varphi$, and offset $B$ to be determined.

$$
\begin{gathered}
A=\frac{2}{N} \sqrt{\left[\Sigma I_{j} \cos \left(j \frac{2 \pi}{N}\right)\right]^{2}+\left[\Sigma I_{j} \sin \left(j \frac{2 \pi}{N}\right)\right]^{2}}, \\
B=\frac{\Sigma I_{j}}{N}, \\
\varphi=a \tan 2\left(\Sigma I_{j} \cos \left(j \frac{2 \pi}{N}\right), \Sigma I_{j} \sin \left(j \frac{2 \pi}{N}\right)\right) .
\end{gathered}
$$

Finally, the range, $r$, is calculated for each pixel from the measured phase by

$$
r=\frac{\varphi c}{4 \pi f}
$$

where $c$ is the speed of light and $f$ is the amplitude modulation frequency of the illumination source and image sensor.

Because of the parallel nature of the imaging sensor, range imaging cameras are inherently suited to producing measurements of an entire scene at rapid update rates. This is particularly desirable for applications such as machine vision, where an object may be moving through the scene. To facilitate this measurement rate, it is desirable to minimize $N$, the number of correlation (intensity) samples captured and read out from the image sensor. As there are three unknowns $(A, B, \varphi)$, the minimum required value of $N$ is also 3 to uniquely determine all unknowns. Typically, however $N=4$ is used to simplify Eqs. (1) and (3), as the sine and cosine terms evaluate to $\{-1,0,1\}$.

\section{Aliasing}

Equations (1) and (3) rely on the assumption that the illumination or image sensor amplitude modulation 
waveforms follow a sinusoidal shape, and the correlation function, therefore, does not contain higher-order harmonics. The illumination source in available cameras typically consists of a bank of infrared LEDs that are amplitude modulated using pulsed (or square) shaped currents [4] to minimize the LED rise and fall times in a manner similar to that described by Schubert [12]. The resultant optical amplitude modulation waveform lies somewhere between a square wave and a sinusoid due to the limited rise and fall times of the LEDs. The image sensor is also typically driven by a digital modulation waveform, producing nonsinusoidal gain modulation $[13,14]$. The combination of these two waveforms produces a correlation function that contains a number of higher frequency harmonic components.

These higher-order harmonics are aliased during sampling. The value of $N$ is akin to the sampling rate of a standard analog-to-digital converter, as can be realized from the illustration in Fig. 1, where four equally spaced samples have been collected over the waveform period for $N=4$. In the presence of higherorder harmonics, the sampling rate (i.e., the value of $N$ ) must be at least twice the highest frequency component contained within the correlation function to fulfill the requirements of the Nyquist-Shannon sampling theorem. If this condition is not met, harmonics of order $h=m N \pm 1$ are aliased onto the signal of interest (the fundamental component), where $m$ is an integer [15]. These aliased harmonics can then have an effect on the phase measurement in Eq. (3). Figure 2 illustrates the normalized frequency spectrum of an illumination source that is amplitude modulated with a square waveform, where the odd-order harmonics (third, fifth, seventh,...) are identified to alias onto the fundamental component of interest for operation with $N=4$ if the image sensor also utilizes square wave modulation. The fundamental frequency component is larger than the DC component, as this figure illustrates only the relative

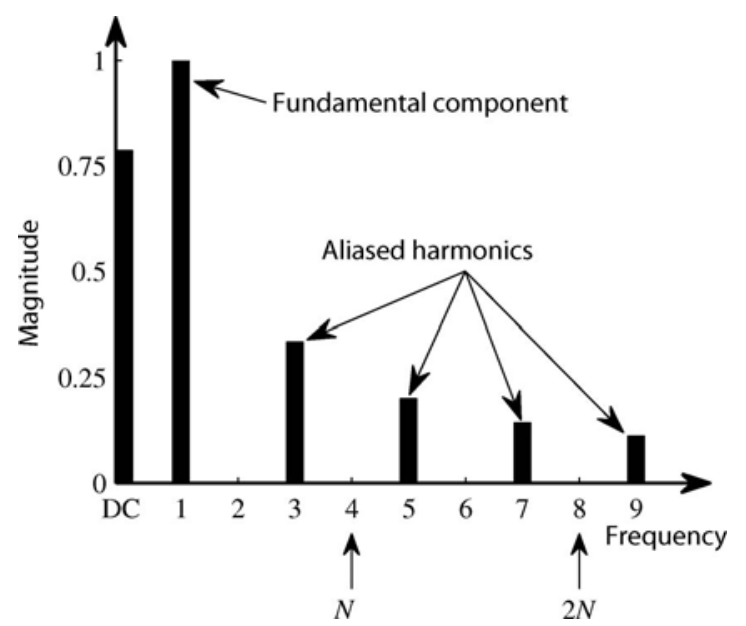

Fig. 2. Normalized spectrum of a square wave generated using an ideal illumination source. The odd-order harmonics are aliased onto the fundamental component during sampling when $N=4$ if the image sensor is also modulated with a square wave. magnitude of each frequency component (and not the corresponding phase) and, therefore, interactions, such as destructive interference, between harmonic components are not apparent.

A simulation of the resultant phase calculated using Eq. (3), plotted against the true phase, is shown in Fig. 3 in the presence of aliased odd harmonics when both the illumination source and the image sensor are amplitude modulated with square waves. Instead of an ideal straight line, a wobblelike feature is seen. The magnitude of the ensuing error, shown in the bottom portion of Fig. 3, is characterized by a distinct oscillation that repeats four times as the true phase transverses from 0 to $2 \pi$ radians.

A calibration routine is typically used to measure this systematic error [1-5], and compensation is applied during postprocessing, for example, by using a lookup table [1] or by fitting functions, such as bsplines [3]. This approach is limited by the accuracy of the calibration parameters, and can be particularly time consuming if independent characterization of each individual image sensor, or even every individual pixel, is required $[3,6]$. If manufacturing parameters are altered, e.g., the illumination source is replaced with a higher power version, or system parameters such as the modulation frequency are modified, the harmonic content of the resultant correlation function is also likely to change, requiring recalibration of the system. Environmental factors, such as temperature changes, can also influence the calibration parameters, resulting in incomplete elimination of the systematic measurement errors.
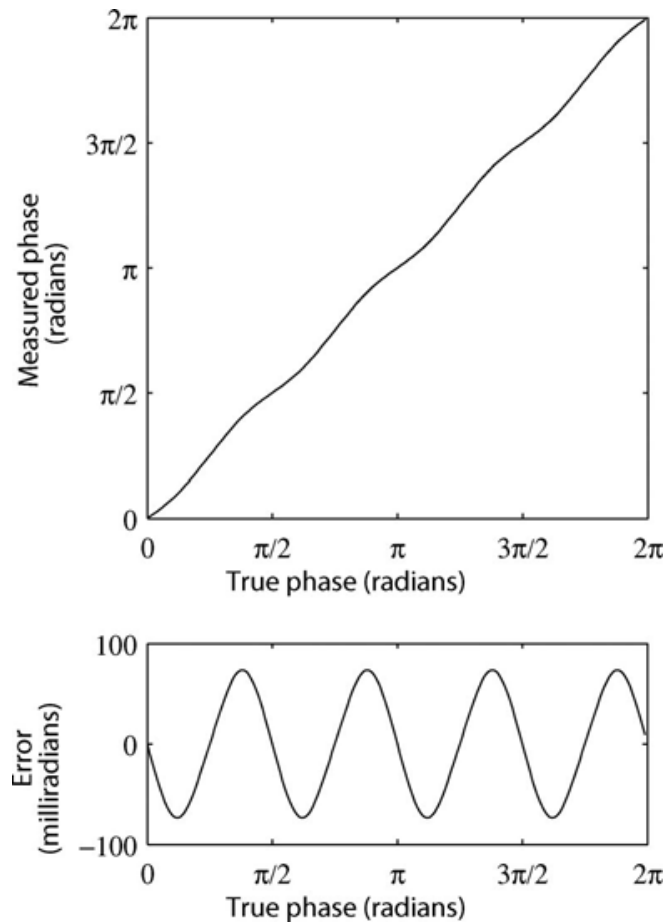

Fig. 3. Above, simulation of the measured phase showing a systematic "wobble" due to aliased harmonics from square wave amplitude modulated illumination and sensor waveforms. Below, the resultant phase error. 
Although Eq. (3) assumes a sinusoidal correlation waveform, it is also possible to calculate the phase shift from other known correlation waveform shapes. A phase determination equation is provided by Lindner et al. [16] for the case where both the illumination source and the image sensor are modulated with square waveforms; hence, the correlation waveform is triangular. In practical systems, the illumination and sensor amplitude modulation waveforms lie somewhere between a square wave and a sinusoid, so the assumption of a triangular correlation function also introduces phase measurement errors. A more general solution proposed by Lindner et al. is to compute the phase shift using a combination of both the triangular- and the sinusoidal-based methods [16]. While this approach was shown to provide some improvement over a single phase measurement, the performance is dependent on the selection of the parameters used to combine the two phase measurements to approximate the true correlation waveform shape. Similar to other calibration techniques, selection of the optimal parameters can be a timeconsuming task, and the phase correction remains valid only if the correlation waveform shape remains constant.

Ideally, instead of attempting to correct for the systematic phase error, the influence of harmonic components should be avoided before or during the sampling process so that the phase measurement is independent of the correlation waveform shape. In analogous electronic systems, this is often achieved by using a low-pass (or anti-aliasing) filter at the input; however, for the range imaging system, no simple equivalent optical filter exists. One simple method of avoiding aliasing is to increase $N$, the number of samples acquired, so that the equivalent sampling rate is above the Nyquist rate and the harmonics present in the correlation function are, therefore, not aliased onto the fundamental signal of interest. This approach provides excellent results [7]; however, as the samples are collected sequentially, assuming a fixed integration period per sample, the total acquisition time scales with the number of samples collected $N$. This approach is, therefore, not well suited to dynamic scenes, where objects are likely to move during the extended acquisition period, causing blurring within the range image. In theory, one could simply reduce the integration period of each sample while collecting a greater number of samples (to maintain a constant total acquisition time). In practice, however, the image sensor readout time and readout noise preclude the use of this approach for large values of $N$.

Another method to avoid the linearity error is to ensure that the correlation function is sinusoidal and, therefore, does not contain harmonics that can be aliased. As harmonics are only present in the correlation function if both the illumination source and the image sensor gain amplitude modulation waveforms contain harmonics of the same order, a simple solution is to ensure that one of these devices comprises only sinusoidal modulation. As described above, LED illumination sources are typically driven with pulsed currents to minimize the rise and fall times (to maximize the permissible modulation frequency) and, in a similar fashion, the image sensor is also typically modulated with digital (square) transistor switching. Achieving sinusoidal modulation therefore becomes a complicated task, especially at high modulation frequencies or if the LEDs and sensor exhibit a nonlinear response. Laser diodes could potentially overcome the amplitude modulation bandwidth restrictions that LEDs face; however, a substantially more complicated electronic drive circuit is required to ensure the laser diode is correctly biased and sinusoidally modulated compared with the simple LED pulsed current drivers currently utilized.

\section{Harmonic Cancellation}

An alternative technique has been developed to avoid the effect of aliased harmonics, where, during the sampling process, phase modulation is applied to the amplitude modulated illumination signal $[17,18]$. A similar concept is used for the generation of lowfrequency signals [19] and also within RF mixer devices [20], where the basic premise is to sum together multiple phase shifted waves of an arbitrary shape (often square waves) with different weightings to approximate a sinusoid.

As illustrated in Fig. 2, odd-order harmonics are aliased onto the fundamental frequency component during standard operation (when the number of samples, $N$, is four), with the third-order harmonic most likely to have the largest amplitude and greatest impact on measurement linearity. We therefore wish to initially cancel this harmonic component during the sampling process. Acquisition of the first sample $I_{0}$ commences with the illumination and image sensor modulation in phase (at $0^{\circ}$ ), and integration begins as per normal operation. The integration time is divided into two equal parts and, for the second half of the integration period, the illumination source amplitude modulation signal is phase shifted by $60^{\circ}$. The phase shift introduced to the illumination source modulation signal produces a corresponding phase shift of the correlation waveform. Therefore, during the integration period, the image sensor sums the correlation waveform with a phase shifted version of itself. For clarity in this paper, any phase shift that occurs in this manner during the sensor integration period is specified in degrees, whereas other phase values are specified in radians.

As the phase shift experienced by the $k$ th-order harmonic is $k$ times that of the fundamental component, for the second half of the integration period, the third-order harmonic is shifted by $180^{\circ}\left(3 \times 60^{\circ}\right)$. Summation of the original waveform with the phase shifted version of itself therefore results in destructive interference for the third-order harmonic, and the effective amplitude modulated illumination waveform as seen by the image sensor, therefore, no longer contains this harmonic component. It 
should be noted that the $60^{\circ}$ phase shift also introduces some amplitude reduction in the fundamental frequency component, which is discussed in more detail in Section 6. The system then continues to acquire the remainder of the $N$ samples, applying the same phase shifting process to each integration period, before finally calculating the range for each pixel in the standard manner [using Eqs. (3) and (4)], while avoiding the linearity error previously caused by the third harmonic.

As there are multiple aliased (odd-order) harmonics in Fig. 2, the technique can be extended to also cancel these additional harmonic components by dividing the integration period into a greater number of segments, with finer phase shift resolution between each segment. To cancel all odd harmonics up to (and including) the $k$ th order requires $n=(k+$ 1)/2 segments, each separated by a phase shift of $180^{\circ} /(n+1)$. A weighting is applied to each segment by dividing the total integration time unevenly between the segments, where the weighting $\alpha_{l}$ of the $l$ th integration segment can be found by dividing the half-sine curve into $n+1$ equal width parts (adapted from [19]):

$$
\alpha_{l}=\sin \frac{l \pi}{n+1},
$$

where $l=1, \ldots, n$. For example, to cancel both the third- and the fifth-order harmonics $(k=5)$ requires $n=3$ segments with phase shift resolution of $45^{\circ}$ and weighting $\alpha=\{1 / \sqrt{ } 2,1,1 / \sqrt{ } 2\}$. This configuration is shown in Fig. 4 for a square-shaped illumination amplitude modulation waveform, where the weighting parameter causes the second (central) segment to extend over a longer time period than the other two segments, while phase shifts of $-45^{\circ}, 0^{\circ}$, and $45^{\circ}$ are applied to each segment, respectively. The phasor diagram in Fig. 5 illustrates the weighting and phase shift applied within the integration period for the fundamental, third-, and fifth-order harmonics; when summed together over the integration period, the third and fifth harmonic components suffer complete destructive interference and are canceled from the resultant waveform. In this instance, the 11th,

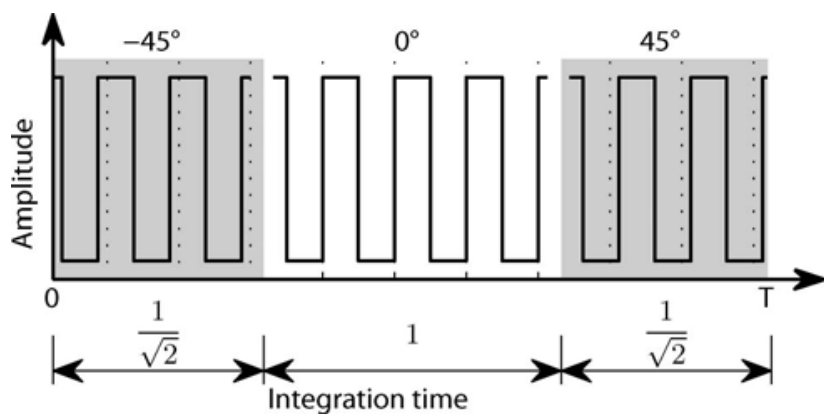

Fig. 4. To cancel the third and fifth harmonics, the sensor integration period $T$ is divided into three segments with a ratio of $1 / \sqrt{ } 2: 1: 1 / \sqrt{ } 2$. The illumination amplitude modulated signal is phase shifted by $-45^{\circ}, 0^{\circ}$, and $+45^{\circ}$ in each segment, respectively.

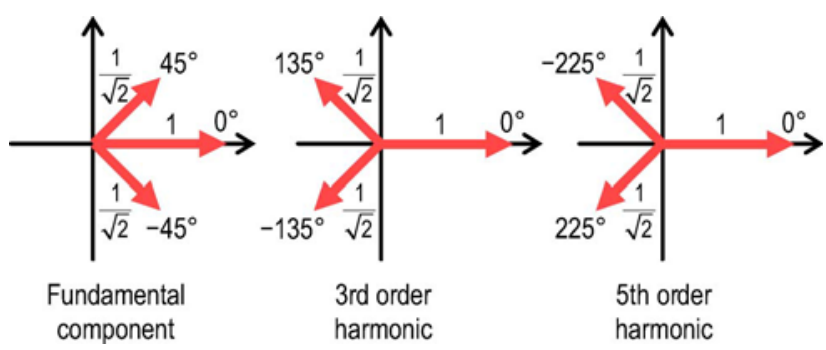

Fig. 5. (Color online) Phase vector diagram of harmonic cancellation using $45^{\circ}$ phase steps. Summation of the third- and fifth-order harmonic components during the image sensor integration period results in complete cancellation due to destructive interference.

13th, 19th, 21st, ..., etc., harmonics are also canceled from the waveform during this process, resulting in the spectra illustrated in Fig. 6 .

To see the improvement in measurement linearity, the simulation in Fig. 3 was repeated, this time applying cancellation of the third- and fifth-order harmonics, with the resultant error shown in Fig. 7. The peak-to-peak error from the original simulation is reduced from 147 to $16 \mathrm{mrad}$. The residual error now shows eight oscillations over the $2 \pi$ phase shift and is primarily caused by aliasing the seventh- and ninth-order harmonics. The technique can be further extended to remove any number of odd-order harmonic components by simply increasing $n$; it is therefore possible to completely eliminate the linearity error from a practical range imaging system.

It is important to note that, although the illumination and sensor amplitude modulation waveforms were assumed to be square in the simulations above, this is not a requirement for harmonic cancellation to be performed. Cancellation is achieved using the same (phase shifted) amplitude modulation waveform to cancel each harmonic with itself; therefore,

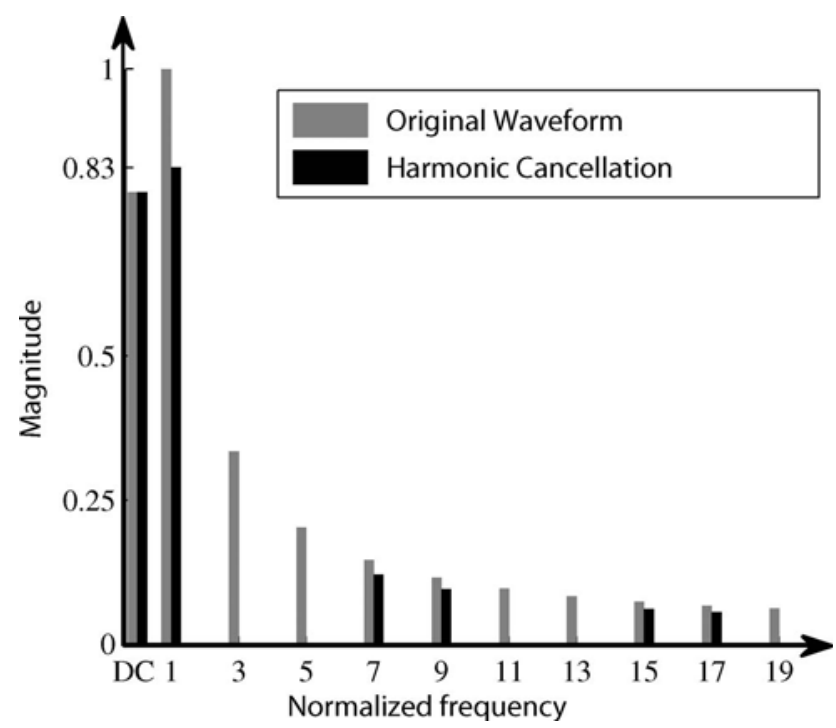

Fig. 6. Spectra of a square wave amplitude modulated illumination signal. Harmonic cancellation has been applied using phase steps of $-45^{\circ}, 0^{\circ}, 45^{\circ}$, and an integration ratio of $1 / \sqrt{ } 2: 1$ : $1 / \sqrt{ } 2$ to remove the third- and fifth (and 11th, 13th, 19th, 21st...)-order harmonics from the waveform. 

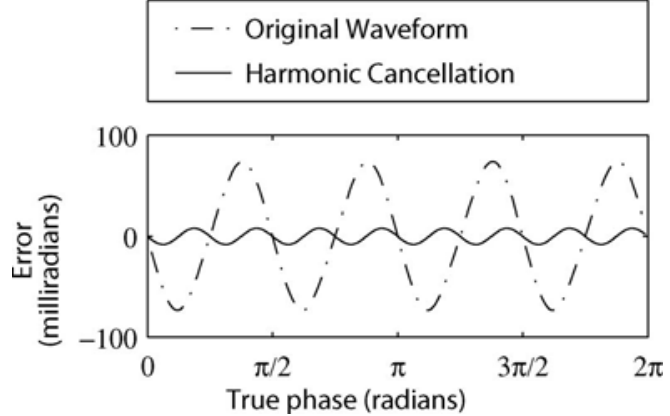

Fig. 7. Simulation of the systematic phase error after the thirdand fifth-order harmonics have been canceled from the square wave amplitude modulated illumination waveform. The error is reduced from 147 to 16 mrad peak to peak compared to standard operation (Fig. 3).

the shape of the waveform is not significant. The only requirement is that the amplitude modulation is invariable during the sensor integration period. Using this harmonic cancellation technique, the linearity error can, therefore, be removed from the phase measurement independent of the actual hardware used for a particular range imaging system. For example, the technique is equally valid for use with LEDs or laser diodes as the illumination source.

\section{Experimental Validation}

The harmonic cancellation technique was implemented by reconfiguring an existing time-of-flight range imaging system [8]. The basic configuration is illustrated in Fig. 8. The system uses an array of four diverging laser diodes to flood-illuminate the scene, which, in this instance, is comprised of a flat white test panel. The existing signal generator (a direct digital synthesizer) used to control the illumination and the image sensor amplitude modulation was replaced with a field programmable gate array (FPGA) (Nios II, Stratix II Development Kit, Altera, San Jose, California, USA) that is capable of generating the modulation signals with preprogrammed phase offsets and durations. A modulation frequency of $66.7 \mathrm{MHz}$ was selected to simplify the generation of the required phase steps within the FPGA.

The light reflected from the scene is imaged by a standard camera lens onto an image intensifier (labeled as "shutter" in Fig. 8) that is used to perform the image sensor gain modulation, also at $66.7 \mathrm{MHz}$.

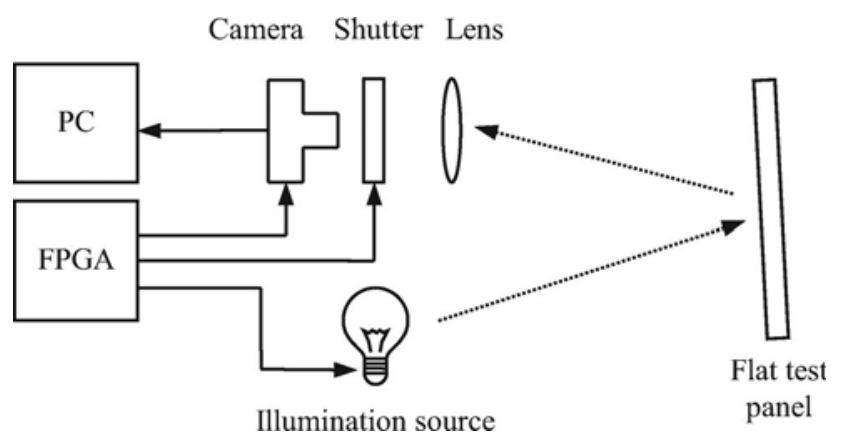

Fig. 8. Implementation of the range imaging system.
The output from the image intensifier is digitized by a CCD camera (Pantera TF 1M60, Dalsa Corporation, Canada) and the data are transferred to a personal computer for processing. Further details about this system are described by Dorrington et al. [8]. Although, in theory, the laser diodes could be amplitude modulated with a sinusoidal response at the required frequency, in practice they are driven by a laser switch integrated circuit (iC-HK, iC Haus, Germany) that simplified the electronic design; therefore, the amplitude modulation waveform contains higher-order harmonic components.

A range image of the flat test panel was acquired by configuring the system to capture four samples for each pixel $(N=4)$, with the resultant phase calculated using Eq. (3). To determine the system's measurement linearity, an electrical time delay of $125 \mathrm{ps}$ was added to the laser illumination signal to emulate the target being shifted to a further distance (instead of actually moving the target). This approach allows the effect of harmonics to be investigated with minimal influence from other possible causes of error, such as the inverse-square relationship between an object's distance and the amount of light that is reflected back to the camera. A second range image is then captured, and another delay of $125 \mathrm{ps}$ is added to the illumination signal. The process is repeated 120 times to produce a series of equally spaced measurements, where the true phase spans 0 to $2 \pi \mathrm{rad}$. The measured phase values for each capture were averaged over a region of $5 \times 5$ pixels near the center of the test panel (to enhance SNR), and then subtracted from the true phase value to produce a graph of the system linearity error in the same manner as the simulation shown in Fig. 3.

Captures were performed with the system operating in a standard configuration; with harmonic cancellation applied to cancel the third and fifth harmonics (using phase steps of $45^{\circ}$ during integration); and with cancellation of practically all interfering odd harmonics (using phase steps of $3^{\circ}$ during integration, canceling harmonics up to the 117th order). The measured linearity error is shown on the left-hand side of Fig. 9 for each of the three cases. Generation of $45^{\circ}$ phase step resolution is simple to implement even with modest hardware (requiring only doubling the signal generator clock) and it provides cancellation of the most dominant third and fifth harmonics, whereas the $3^{\circ}$ resolution was chosen to demonstrate the cancellation of all odd harmonics present in the modulation waveforms, and was dictated by the resolution limit of the phase locked loop within the FPGA. Other phase step resolutions were also tested but are not shown here.

The linearity error was again simulated in a similar fashion to that shown in Fig. 3. Previously, the illumination and sensor were assumed to be amplitude modulated with a square response, but to generate a more accurate simulation, the illumination amplitude modulation waveform was measured directly using a photodiode (DET210, Thorlabs Inc., 

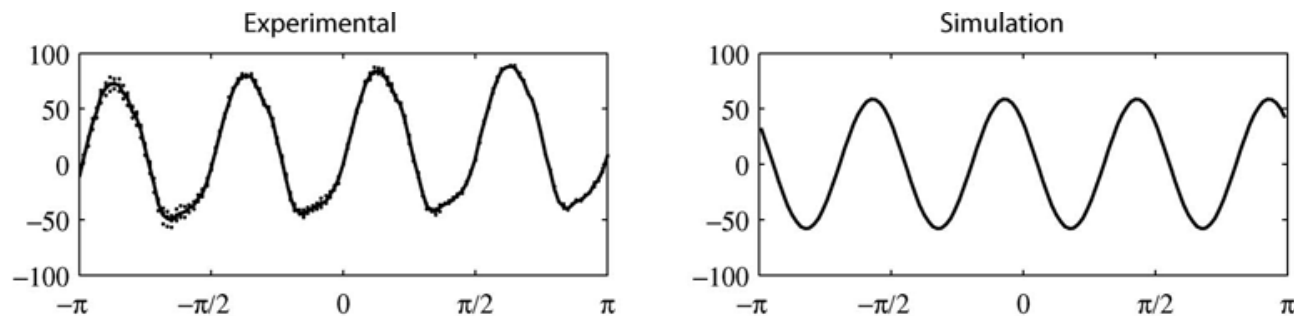

(a)
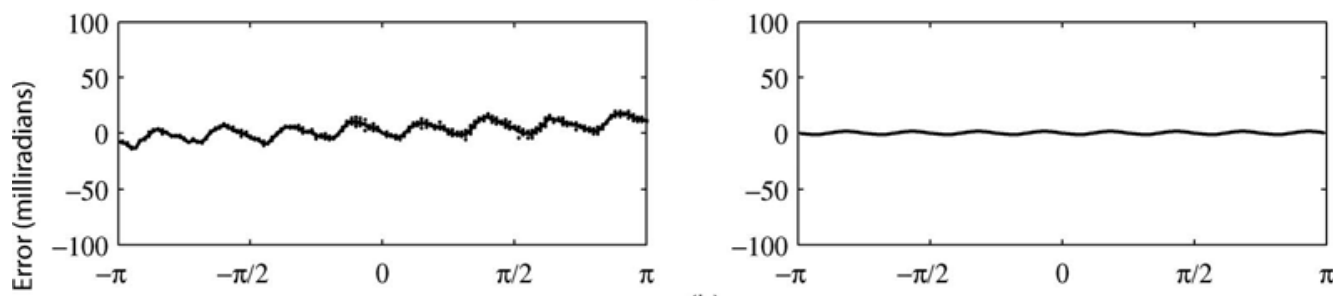

(b)
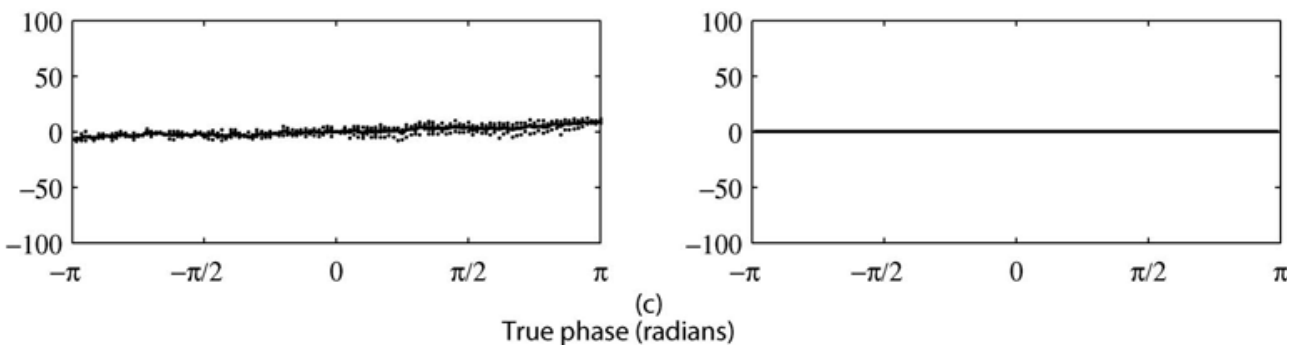

Fig. 9. Simulated and measured linearity error for the range imaging system under (a) standard operation with square wave modulation, (b) with harmonic cancellation of the third- and fifth-order harmonics, and (c) with harmonic cancellation of all odd harmonics (up to the 117th order). Each measured experimental capture was repeated five times (black dots), with the mean value plotted as a solid line.

New Jersey, USA) and the image sensor gain modulation was measured using a technique utilizing a picosecond pulsed laser to optically probe the sensor [14]. The simulation results are shown on the righthand side in Fig. 9.

For the standard method of operation, Fig. 9(a), a linearity error of approximately $130 \mathrm{mrad}$ peak to peak was measured. This error is reduced to $18 \mathrm{mrad}$ peak to peak using harmonic cancellation with phase steps of $45^{\circ}$ to remove the third- and fifth-order harmonics. The remaining error is primarily due to the seventh- and ninth-order harmonics, which produce an eight-cycle oscillation over the $2 \pi$ range, as seen in Fig. 9(b). The error also appears larger in the experimental measurement than it does in the simulation. The illumination amplitude modulation waveform used for the simulation was measured using a DET210 photodiode; this photodiode is likely to partially attenuate the higher-order harmonics due to its limited ( $1 \mathrm{~ns})$ rise time, which is consistent with the reduction of the simulated systematic error. The error contribution due to the third- and fifthorder harmonics (an oscillation of four cycles over the $2 \pi$ range) is less than $2 \mathrm{mrad}$, which was determined by performing a fast Fourier transform on the experimental error in Fig. 9(b) to separate the errors generated by different harmonic components. Finally, with all the odd harmonics up to the 117th order canceled in Fig. 9(c), no oscillation exists in either the experimental or simulated data.
The experimental and simulated data have different offsets on the $x$ axis due to various fixed time delays within the system. The experimental graphs also show a linear trend of approximately $16 \mathrm{mrad}$ over the capture time that is likely due to heating the image intensifier (and driver circuit) and is independent of the systematic linearity error introduced by aliased harmonics. Further investigation is being performed to mitigate this error source.

\section{Signal Amplitude}

The standard deviation of range measurements $\sigma_{R}$ for an ideal system can be expressed as [9]

$$
\sigma_{R}=\frac{c}{4 \pi f \sqrt{2}} \cdot \frac{\sqrt{B}}{c_{\mathrm{demod}} B_{\mathrm{sig}}}
$$

where $B$ is the signal offset, which comprises two parts: an offset due to ambient light $\left(B_{\mathrm{amb}}\right)$ and an offset due to the DC component of the signal itself $\left(B_{\text {sig }}\right)$. The relationship between these terms can be seen in Fig. 1. The final term, $c_{\text {demod }}$, is the (de)modulation contrast, which is defined as the measured signal amplitude $A$ divided by the measured signal DC component $B_{\text {sig }}$ (the offset when no ambient light is present).

For the case of a nonsinusoidal correlation waveform, the measured signal amplitude $A$ calculated using Eq. (1) becomes phase dependent due to 
harmonics being aliased constructively, or destructively, on to the fundamental component. As the measured DC component $B_{\text {sig }}$ remains constant, the demodulation contrast (hence, the range measurement standard deviation) exhibits this same phase dependency. In practical terms, this means that range measurement precision is dependent on an object's range (in addition to other parameters, such as the modulation frequency, object color, and reflectivity, and the level of ambient light in the scene).

The demodulation contrast was measured using the previously captured experimental data and is shown in Fig. 10. For standard operation, the measured demodulation contrast contains a systematic oscillation varying between approximately 0.76 and 0.88 with a mean value of 0.83 . In comparison, with harmonic cancellation applied using a $3^{\circ}$ phase step resolution, the aliased harmonics are removed during sampling and the demodulation contrast remains almost constant over the entire input phase range (a peak-to-peak variation of 0.015 was measured). However, as can be seen in Fig. 10, applying the harmonic cancellation technique also decreases the demodulation contrast value, with the mean value reduced to 0.65 .

The attenuation of the demodulation contrast is due to the fundamental signal component being partially out of phase with itself during each of the integration period segments, as illustrated in Fig. 5. For harmonic component of order $h$, the reduction of amplitude due to applying harmonic cancellation can be calculated as

$$
\Delta A_{h, n}=\frac{\left|\sum_{l=1}^{n} \alpha_{l} \exp [i l h \pi /(n+1)]\right|}{\sum_{l=1}^{n} \alpha_{l}}
$$

where $\alpha_{l}$ is the weighting of each integration segment from Eq. (5), and $n$ is the total number of integration segments used. For the case of $n=3$ with $45^{\circ}$ phase

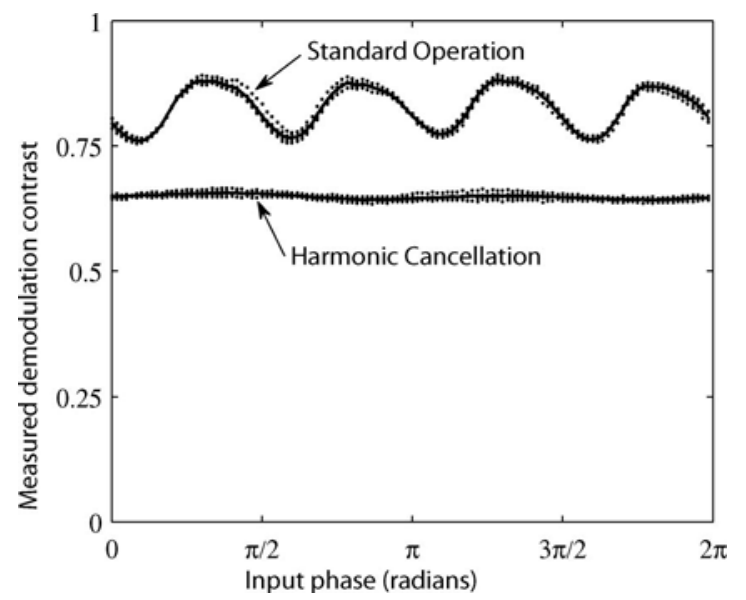

Fig. 10. Comparison between the measured demodulation contrast for standard operation and the harmonic cancellation technique applied with $3^{\circ}$ phase step resolution. Each measurement was repeated five times (black dots), with the mean value plotted as a solid curve. step resolution, the amplitude of the third and fifth harmonic components $(h=3,5)$ is reduced to zero, while the amplitude of the fundamental component $(h=1)$ is reduced to $(2 \sqrt{ } 2) /(2+\sqrt{ } 2)=0.83$ of the original amplitude. These changes in amplitude can also be seen in Fig. 6. As the technique is extended to cancel a greater number of odd-order harmonics, such as that experimentally demonstrated in Section 5 to the 117th order, from Eq. (7) the change in amplitude of the fundamental component approaches $\pi / 4=0.79$.

The DC component of the signal, $B_{\text {sig }}$, remains constant when harmonic cancellation is applied (as shown in Fig. 6); hence, the mean demodulation contrast value will be reduced proportionally with the amplitude of the fundamental component. The range measurement standard deviation is inversely proportional to the demodulation contrast $c_{\text {demod }}$, as given by Eq. (6), and as a result the mean range measurement standard deviation is expected to increase when harmonic cancellation is applied. From the experimental data shown in Fig. 10, the measured change of the mean values of $c_{\text {demod }}$ when harmonic cancellation is applied is $0.65 / 0.83=0.78$, which is in close agreement with the predicted value of $\pi / 4=0.79$. The mean standard deviation is, therefore, expected to increase by $1 / 0.78=1.28$.

\section{Experimental Precision Measurement}

To experimentally verify any change in measurement precision, a range image was acquired using the flat panel target, which was then divided into approximately 200 regions of $5 \times 5$ pixels. Since the target is known to be planar, the pixel range values for each region were fit to a reference plane, and the 1sigma standard deviation of the residuals measured for each region. The mean offset value $B_{\text {sig }}$ was also recorded for each region as the measurement precision is dependent on the signal intensity as given by Eq. (6). The measurement was performed with the same scene both for standard operation, and with harmonic cancellation using phase steps of $3^{\circ}$. As the measurement precision is phase dependent in the presence of aliased harmonics (due to the varied measured demodulation contrast shown in Fig. 10), an electronic delay was again added to the light source signal and the precision measurements were repeated 120 times for different phase values spanning 0 to $2 \pi \mathrm{rad}$. The results shown in Fig. 11 are the mean standard deviation value measured for each region in the scene over the entire phase sweep. It has been assumed that, by segmenting the range image into small regions, systematic distortions (such as lens distortions) are negligible and can, therefore, be ignored for this precision measurement. At the very least, any distortions are constant for both captures.

There are two effects that can clearly be seen in Fig. 11; first, the measurement precision is dependent on the intensity of the modulated light reaching each pixel, where brighter regions within the scene 


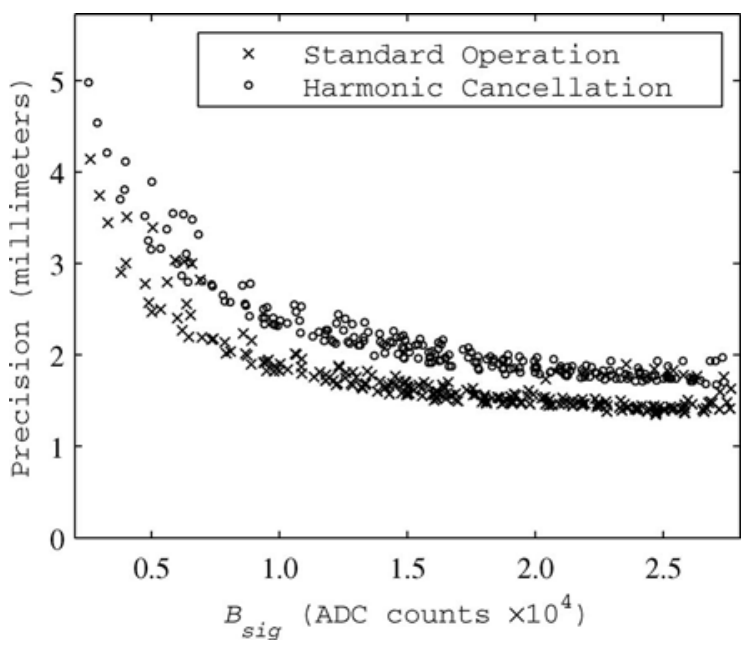

Fig. 11. Measured precision ( $1 \sigma$ standard deviation) versus measured offset $B_{\text {sig }}$ for approximately 200 regions within a range image. The error is higher for the harmonic cancellation technique compared to standard operation. The offset values are measured in $\mathrm{ADC}$ counts from the camera.

(corresponding to a larger $B_{\text {sig }}$ value) result in a reduction of the standard deviation, and, second, the measurement precision is worse when harmonic cancellation is implemented. In this instance, when harmonic cancellation was applied, the standard deviation of the analyzed regions in the scene increased by a mean value of $1.24 \pm 0.05$ times that of standard operation. While Eq. (6) models an ideal system that is limited by photon shot noise (other noise sources, such as dark current and noise within the image intensifier, etc., are not included), the experimentally measured change in precision caused by the reduction of demodulation contrast is consistent with the predicted value of 1.28 .

The linearity error measured in Fig. 9 is approximately 130 mrad peak to peak for the standard homodyne method using square wave modulation, which corresponds to a peak-to-peak error of approximately $47 \mathrm{~mm}$ for the $66.7 \mathrm{MHz}$ modulation frequency used [from Eq. (4)]. In Fig. 11 the measured precision (standard deviation) is typically of the order of $2-3 \mathrm{~mm}$, dependent on the intensity of the received light, which is more than 1 order of magnitude better than the system accuracy. Therefore, trading a relatively small reduction in measurement precision for a significant improvement in measurement accuracy (linearity) is likely to be acceptable for most applications.

\section{Increasing the Demodulation Contrast}

Range measurement precision is inversely proportional to the demodulation contrast value $c_{\text {demod }}$ as given by Eq. (6); hence, it is desirable to maximize this value where possible. Lange [1] describes the case where the illumination source is modulated with a sinusoidal waveform, while the image sensor is modulated with a square wave with $50 \%$ duty cycle, resulting in a maximum possible demodulation contrast value of $\operatorname{sinc}(0.5)=0.64$, where the term $\operatorname{sinc}(x)$ is used to refer to the normalized sinc func- tion, i.e., $\operatorname{sinc}(x)=\sin (\pi x) /(\pi x)$. Similarly, a maximum demodulation contrast of 0.90 can be achieved if the image sensor is modulated with a $25 \%$ duty cycle rectangular waveform $[1,10]$.

For the range imaging system utilized here, it is possible to modulate the shutter with a $25 \%$ duty cycle waveform; however, this comes at the expense of requiring driver electronics with higher bandwidth. Also, the amount of reflected light collected by the camera is halved since the shutter period is also halved (compared to normal 50\% duty cycle operation), resulting in a reduction of range measurement precision.

Specialized CMOS/CCD range imaging sensors are typically capable of collecting two samples simultaneously that are $180^{\circ}$ out of phase (known as twotap pixels) $[6,9,11,21]$, for example, $I_{0}$ and $I_{2}$ in Fig. 1. This improves the efficiency of the system as all the light reflected from the scene is collected by the image sensor (as opposed to the hardware used here, which discards the light during the off period). Similarly, specialized image sensors have been produced where each pixel collects and stores four samples simultaneously (known as four-tap pixels) $[1,10,22]$, again still collecting all the reflected light from the scene, but in this instance with $25 \%$ duty cycle gain modulation. These systems can, therefore, (theoretically) produce higher demodulation contrast values up to 0.90 . The four-tap pixel design increases the complexity of each pixel, however, usually with a corresponding reduction of fill factor; therefore, twotap pixels with $50 \%$ duty cycle operation are typically used in actual systems [9].

As the sampled waveform is a correlation between the illumination and the image sensor modulation waveforms, instead of requiring a four-tap image sensor, the same increase in demodulation contrast can be achieved by utilizing a $25 \%$ modulation duty cycle for the illumination source. In fact, for an arbitrary duty cycle rectangular waveform, the illumination waveform contributes to the demodulation contrast as sinc (duty cycle), as shown in Fig. 12.

Under normal operating conditions, changing the illumination duty cycle also increases the harmonic content within the waveform, which is likely to exaggerate the range measurement linearity problem discussed above. However, by concurrently applying the harmonic cancellation technique, the duty cycle of the illumination source can be altered while mitigating the disruptive effects of any increase in harmonic content. It is, therefore, possible to overcome the decrease in demodulation contrast generated by use of the harmonic cancellation technique, and to even exceed the mean performance of the standard system by selecting a short duty cycle for the illumination source.

The demodulation contrast was measured using the range imaging system, again configured with harmonic cancellation with a step size of $3^{\circ}$ during the integration period. In this instance, the laser amplitude modulation waveform was also modified, 


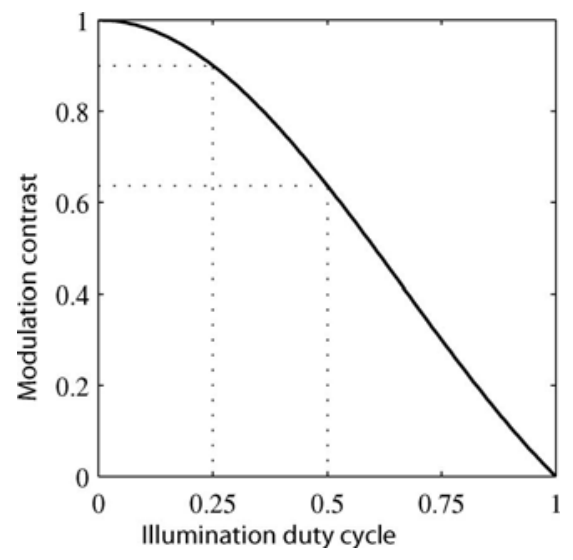

Fig. 12. Reducing the duty cycle of the amplitude modulated illumination waveform results in an increase of the modulation contrast. A typical duty cycle of $50 \%$ contributes a modulation contrast of 0.64 , while a value of 0.90 can be achieved by reducing the duty cycle to $25 \%$.

being set to an approximately rectangular shape with duty cycles of $38 \%, 20 \%$, and $3 \%$ (as measured with a photodiode, DET210, Thorlabs Inc., New Jersey, USA). The correlation function is shown in Fig. 13 as the phase was again electronically shifted from 0 to $2 \pi \mathrm{rad}$. The demodulation contrast was measured to be 0.69 at $38 \%, 0.82$ at $20 \%$, and 0.94 at $3 \%$ duty cycle. As expected, the shorter duty cycle produces a high demodulation contrast value, while the correlation function maintains a near-sinusoidal shape for all three cases due to the cancellation of the odd-order harmonics.

Reducing the duty cycle of the illumination source to improve the demodulation contrast value creates a new problem; the average optical power emitted by the system is decreased. The experimental setup used to capture these data includes an automatic gain feature to ensure an optimal setting for each capture. This means each plot in Fig. 13 has a similar amplitude despite the reduction of illumination power. It should be noted that this gain does not alter the demodulation contrast value, as both the amplitude and the offset are scaled proportionally to one another. However, for the practical purpose of capturing range images, the measurement precision decreases due to the reduction of optical power.

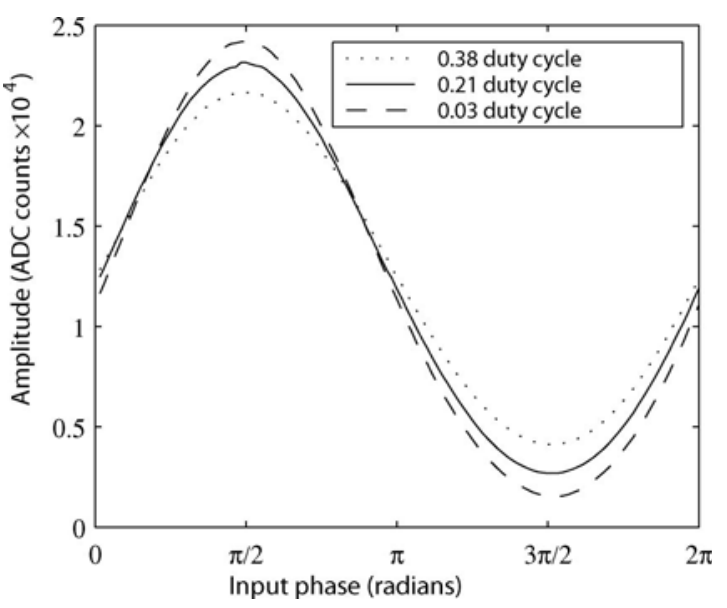

Fig. 13. Measured correlation function for different laser duty cycles. The shortest duty cycle produces the largest demodulation contrast (largest peak-to-peak amplitude). All odd-order harmonics were canceled during sampling, so the waveform appears approximately sinusoidal regardless of the laser duty cycle used.

A simple solution to this problem is to maintain the average optical power by increasing the peak power of the amplitude modulated illumination source. For example, the range imaging system above uses four laser diodes (ML120G21, Mitsubishi Electric Corp., Japan), which are rated by the manufacturer for (continuous wave) operation with an optical power of $80 \mathrm{~mW}$ per diode, which is achieved by setting the peak power to $160 \mathrm{~mW}$ for standard $50 \%$ duty cycle square wave amplitude modulation. By reducing the duty cycle to $25 \%$ while also increasing the peak optical power to $320 \mathrm{~mW}$, the range imaging system maintains the same average optical power, and the range measurement precision is improved (due to the increased demodulation contrast). Unfortunately, this peak power requirement exceeds the manufacturer's rating for the laser diodes currently being used ( $160 \mathrm{~mW}$ for pulsed operation). Two possible solutions for this system are to either increase the size of the laser diode array, for example, to eight laser diodes instead of four, each operating with a lower average power; and/or to use laser diodes with a higher peak power rating. Advances in laser diode technology continue to provide devices with in-

Table 1. Demodulation Contrast Values for Various Image Sensor and Illumination Amplitude Modulation Waveforms, for Standard Operation, and Operation with Harmonic Cancellation

\begin{tabular}{cclcc}
\hline Image Sensor Modulation & Illumination Modulation & Sampling Technique & Demodulation Contrast & Aliased Harmonics \\
\hline Sine & Sine & Standard operation & 0.50 & None \\
Square & Sine & Standard operation & 0.64 & None \\
Square & Square & Standard operation & $\mathbf{0 . 8 1}^{b}$ & Present \\
Square & Square & Harmonic cancellation & 0.64 & None \\
Square & $35 \%$ duty cycle & Harmonic cancellation & 0.81 & None \\
Square & $25 \%$ duty cycle & Harmonic cancellation & 0.90 & None \\
Square & $1 \%$ duty cycle & Harmonic cancellation & 1.00 & None \\
\hline
\end{tabular}

\footnotetext{
${ }^{a}$ The line in bold highlights the typical configuration used in existing systems; $50 \%$ duty cycle square wave amplitude modulation for the image sensor and illumination source.

${ }^{b}$ Aliased harmonics cause the demodulation contrast value to vary between 0.707 and 1.00 dependent on the input phase. The mean value of 0.81 is used for comparison with the other techniques that do not suffer from this phase dependency.
} 
creased power ratings; for example, the existing diodes could be replaced with ML101J27 (Mitsubishi Electric Corp., Japan) laser diodes, which are rated for $130 \mathrm{~mW}$ continuous operation, and $350 \mathrm{~mW}$ pulsed operation.

Briefly ignoring the technical difficulties of maintaining a constant average optical power, in particular for very high peak power and short duty cycle situations, Table 1 gives the theoretical demodulation contrast value for a number of different combinations of image sensor and illumination amplitude modulation. As currently available range imaging cameras typically utilize square wave modulation for the image sensor and illumination source, this row is indicated in bold in Table 1 and is used for comparison purposes. It can be seen that applying harmonic cancellation reduces the mean demodulation contrast value, in this case from 0.81 to 0.64 . By reducing the illumination duty cycle to $35 \%$ (while the image sensor retains square modulation), the demodulation contrast value is restored to 0.81 , while ensuring the correlation function no longer contains harmonics.

Continued reduction of the illumination duty cycle (less than 35\%), combined with harmonic cancellation, further improves the demodulation contrast while also avoiding aliased harmonics. A suggested optimal configuration is to use harmonic cancellation with an illumination duty cycle of $25 \%$. Compared to the typical configuration, the illumination modulation bandwidth and peak power requirements are doubled; however, the theoretical demodulation contrast value is increased to 0.90 . It is, therefore, possible to enhance the mean range measurement precision by approximately $11 \%$ compared to the standard configuration, and also improve the range measurement accuracy.

\section{Conclusion}

The performance of AMCW time-of-flight range imaging systems is often constrained by a nonlinear systematic range measurement error. This error is caused by high-order harmonics in the illumination and sensor amplitude modulation waveforms that are aliased during the sampling process. Typically, a calibration is performed to measure and correct this systematic error; however, changes in system parameters (such as the modulation frequency) or environmental conditions (such as temperature) can invalidate this calibration.

An alternative sampling technique overcomes this limitation by canceling the interfering harmonics during the sampling process. The main advantage gained using this approach is that the cancellation is independent of the amplitude modulation waveform shape, so there is no need to measure the system response or to recalibrate the system if the response changes. Experimental results demonstrate the effectiveness of this technique on a practical range imaging system, removing the systematic (oscillatory) error from the measurements.
The disadvantage of applying harmonic cancellation is that the demodulation contrast is reduced, which reduces measurement precision. In some instances/applications, this trade-off may be acceptable; however, experimental results show that, by reducing the duty cycle of the illumination amplitude modulation waveform, the demodulation contrast can be increased. Calculations show that if a constant average optical power is maintained, an illumination source operating with a duty cycle of $35 \%$ (and with harmonic cancellation applied) will achieve a mean measurement precision identical to the standard homodyne method of operation with square wave amplitude modulation. If the illumination duty cycle is further reduced to, for example, $25 \%$, then the range measurement precision will be improved.

By combining harmonic cancellation with a reduced illumination duty cycle, it is, therefore, possible to generate range measurements with both enhanced accuracy (linearity) and precision compared to existing systems. Importantly, this improvement can be achieved without requiring modification of the operation of the specialized image sensor and, therefore, it can easily be applied to existing commercial range imaging cameras.

\section{References}

1. R. Lange, " $3 \mathrm{D}$ time-of-flight distance measurement with custom solid-state image sensors in CMOS/CCD-technology," Ph.D. dissertation (University of Siegen, 2000).

2. T. Kahlmann and H. Ingensand, "High-precision investigations of the fast range imaging camera SwissRanger," Proc. SPIE 6758, 67580J (2007).

3. M. Lindner and A. Kolb, "Lateral and depth calibration of PMD-distance sensors," in Advances in Visual Computing, Part II, Vol. 4292 of Lecture Notes in Computer Science (Springer, 2006), pp. 524-533.

4. C. Niclass, C. Favi, T. Kluter, F. Monnier, and E. Charbon, "Single-photon synchronous detection," IEEE J. Solid-State Circuits 44, 1977-1989 (2009).

5. H. Rapp, M. Frank, F. A. Hamprecht, and B. Jahne, "A theoretical and experimental investigation of the systematic errors and statistical uncertainties of time-of-flight-cameras," IJISTA 5, 402-413 (2008).

6. S. Hsu, S. Acharya, A. Rafii, and R. New, "Performance of a time-of-flight range camera for intelligent vehicle safety applications," in Advanced Microsystems for Automotive Applications 2006 (Springer, 2006), pp. 205-214.

7. A. A. Dorrington, M. J. Cree, D. A. Carnegie, A. D. Payne, R. M. Conroy, J. P. Godbaz, and A. P. P. Jongenelen, "Video-rate or high-precision: a flexible range imaging camera," Proc. SPIE 6813, 681307 (2008).

8. A. A. Dorrington, M. J. Cree, A. D. Payne, R. M. Conroy, and D. A. Carnegie, "Achieving sub-millimetre precision with a solidstate full-field heterodyning range imaging camera," Meas. Sci. Technol. 18, 2809-2816 (2007).

9. B. Büttgen and P. Seitz, "Robust optical time-of-flight range imaging based on smart pixel structures," IEEE Trans. Circuits Syst. I 55, 1512-1525 (2008).

10. B. Büttgen, T. Oggier, R. Kaufmann, P. Seitz, and N. Blanc, "Demonstration of a novel drift field pixel structure for the demodulation of modulated light waves with application in threedimensional image capture," Proc. SPIE 5302, 9-20 (2004). 
11. R. Schwarte, "Breakthrough in multichannel laser-radar technology providing thousands of high-sensitive lidar receivers on a chip," Proc. SPIE 5575, 126-136 (2004).

12. E. Schubert, Light-Emitting Diodes (Cambridge U. Press, 2006).

13. A. D. Payne, A. A. Dorrington, M. J. Cree, and D. A. Carnegie, "Characterization of modulated time-of-flight range image sensors," Proc. SPIE 7239, 723904 (2009).

14. A. D. Payne, A. A. Dorrington, M. J. Cree, and D. A. Carnegie, "Characterizing an image intensifier in a full-field range imaging system," IEEE Sens. J. 8, 1763-1770 (2008).

15. A. A. Dorrington, M. J. Cree, D. A. Carnegie, and A. D. Payne, "Selecting signal frequencies for best performance of Fourierbased phase detection," in Proceedings of Twelfth New Zealand Electronics Conference (Manukau Institute of Technology, 2005), pp. 189-193.

16. M. Lindner, A. Kolb, and T. Ringbeck, "New insights into the calibration of ToF-sensors," in Proceedings of IEEE Conference on Computer Vision and Pattern Recognition (IEEE, 2008), pp. $1-5$.
17. A. D. Payne, A. A. Dorrington, M. J. Cree, and D. A. Carnegie, "Improved linearity using harmonic error rejection in a fullfield range imaging system," Proc. SPIE 6805, 68050D (2008).

18. A. D. Payne and A. A. Dorrington, "Signal simulation apparatus and method," patent WO 2009/051499 (23 April 2009), http://www.wipo.int/pctdb/en/wo.jsp?WO=2009051499.

19. A. C. Davies, "Digital generation of low-frequency sine waves," IEEE Trans. Instrum. Meas. 18, 97-105 (1969).

20. J. A. Weldon, R. S. Narayanaswami, J. C. Rudell, L. Lin, M. Otsuka, S. Dedieu, L. Tee, K. C. Tsai, C. W. Lee, and P. R. Gray, "A $1.75 \mathrm{GHz}$ highly integrated narrow-band CMOS transmitter with harmonic-rejection mixers," IEEE J. Solid-State Circuits 36, 2003-2015 (2001).

21. Z. Xu, R. Schwarte, H. Heinol, B. Buxbaum, and T. Ringbeck, "Smart pixel-photonic mixer device (PMD)," in Proceedings of M2VIP '98-International Conference on Mechatronics and Machine Vision in Practice (1998), pp. 259-264.

22. T. Spirig, M. Marley, and P. Seitz, "The multitap lock-in CCD with offset subtraction," IEEE Trans. Electron Devices 44, 1643-1647 (1997). 